Johanna Patricia A. Cañal, MD, MHA

Department of Radiology

College of Medicine - Philippine General Hospital

University of the Philippines Manila
Correspondence: A/Prof. Dr. Johanna Patricia A. Cañal

Department of Radiology

Philippine General Hospital

Taft Ave., Ermita Manila 1000

Philippines

Phone: $02-5548471$

Fax: $02-5226597$

E-mail: joie_canal@yahoo.com

Reprints will not be available from the authors.

The authors declared that this represents original material that is not being considered for publication or has not been published or accepted for publication elsewhere, in full or in part, in print or electronic media; that the manuscript has been read and approved by all the authors, that the requirements for authorship have been met by each author, and that each author believes that the manuscript represents honest work

Disclosures: The authors signed disclosures that there are no financial or other (including personal) relationships, intellectual passion, political or religious beliefs, and institutional affiliations that might lead to a conflict of interest.

\section{Dermal Metastasis from Nasopharyngeal Carcinoma: A Rare Form of Metastasis}

\author{
ABSTRACT \\ creeping form of dermal metastasis.

\section{Methods:} \\ Design: Case Report \\ Setting: Tertiary Public University Hospital \\ Patient: One
}

Objective: To report a rare form of metastasis from a primary nasopharyngeal carcinoma, a

Results: A 47-year-old male referred for radiotherapy after having undergone a selective neck dissection for multiple cervical lymphadenopathy with histopathologic diagnosis of undifferentiated carcinoma and no known primary underwent a four-quadrant nasopharyngeal biopsy which confirmed the presence of nasopharyngeal carcinoma.

Subsequent radiotherapy resolved the primary mass, and a new posterior cervical lymph node that appeared five months after completion of radiotherapy also resolved with additional radiotherapy. He was asymptomatic for two years until he noted thickening of the skin in his left supraclavicular area. A Computed Tomography (CT) scan showed deep cervical adenopathy and skin thickening, and biopsy confirmed dermal metastatic carcinoma. Two courses of radiotherapy to the affected skin and left axilla where a lymph node had developed resulted in resolution and he was referred for chemotherapy.

Conclusion: Dermal metastasis from nasopharyngeal carcinoma is rare and does not present with pathognomonic symptomatology. It may therefore be confused for a benign side effect (dermatitis), not the malignant manifestation that forebodes a bad prognosis. Patients with dermal metastasis should receive treatment, and radiotherapy may play a significant part. Chemotherapy may also play a role in its management.

Keywords: Nasopharyngeal carcinoma, dermal metastasis

Nasopharyngeal carcinoma is fairly common in Asia. It is said to be endemic among the Southern Chinese population. ${ }^{1,2}$ Standard texts list the most common site of distant metastasis as bone followed closely by lung and liver metastasis ${ }^{1,2,3}$ with hardly any mention of skin metastasis. Skin and dermal metastasis is commonly mentioned in association with breast cancer but not with nasopharyngeal carcinoma. This case presents an unusual occurrence of dermal metastasis in nasopharyngeal carcinoma. 


\section{CASE REPORT}

A 47-year-old married male was first referred to the Philippine General Hospital for radiotherapy and further management in October 2002 following a selective neck dissection for multiple cervical lymphadenopathy in September 2002 with a histopathologic diagnosis of undifferentiated carcinoma. No primary site was identified at the time.

A CT scan of the neck and nasopharynx revealed only cervical lymphadenopathy in the left anterior $(4.2 \times 2.3 \mathrm{~cm})$ and posterior $(4 \times 2.7 \mathrm{~cm})$ triangles. A chest $\mathrm{x}$-ray was normal. No demonstrable nasopharyngeal mass was seen. Because of a high index of suspicion, a four-quadrant nasopharyngeal biopsy was done confirming the presence of undifferentiated carcinoma. The diagnosis was nasopharyngeal carcinoma T1 N1 Mx, Stage IIB.

He underwent radiotherapy to the nasopharynx and neck from December 2002 to February 2003 receiving 76 Gy to the primary site via opposing lateral fields and $50.4 \mathrm{~Gy}$ to the neck via a single anterior low-neck field with a midline block.

He remained asymptomatic for five months before noting new posterior cervical lymph nodes in the nape area. A biopsy again revealed metastatic undifferentiated carcinoma. The previously-untreated posterior neck was subjected to small field radiotherapy delivering 50 Gy over 25 days in June and July 2003.

A repeat CT scan of the nasopharynx and neck in September 2003 revealed no nasopharyngeal mass. The previously-noted cervical lymph nodes in levels II and V decreased in size. The patient reported eventual disappearance of these lymph nodes and he was apparently well for the rest of 2003 and throughout 2004. A follow-up CT scan in June 2004 showed no nasopharyngeal mass but did show enlarged level III deep cervical and posterior cervical lymph nodes on the right. The patient did not present himself for treatment at the time.

Over a year later, in August 2005, he consulted his attending physician for perceived thickening of the skin in his left supraclavicular area. The working impression was radiation dermatitis but another CT Scan was requested which still showed no nasopharyngeal recurrence but revealed deep cervical lymphadenopathy in the left (Figure 1).

On examination, the skin over the left supraclavicular area was erythematous, swollen and had verrucous, plaque-like texture. The lesion involved the left upper chest and neck and crossed the midline (Figure 2). There was some limitation of shoulder movement because of the swelling over the shoulder. A clavicle and shoulder $\mathrm{x}$-ray showed intact osseous structures and skin thickening of the supraclavicular tissues with faint internal lucencies. He was referred to a dermapathologist who did an incisional skin biopsy which revealed metastatic carcinoma (Figure 3).

Another CT scan of the neck in November 2005 revealed enlarged

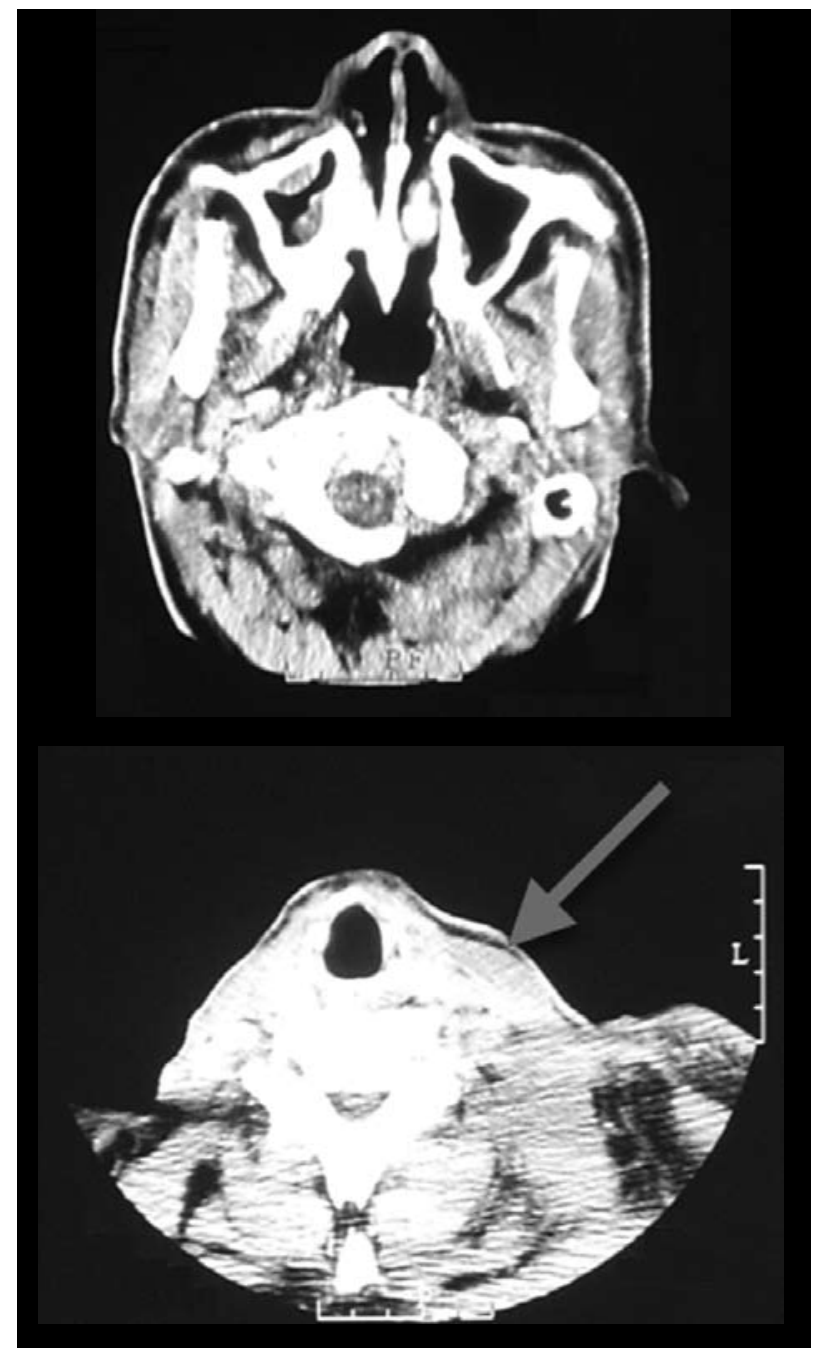

Figure 1. CT scan showing a clear nasopharynx and enlarged left deep cervical nodes.

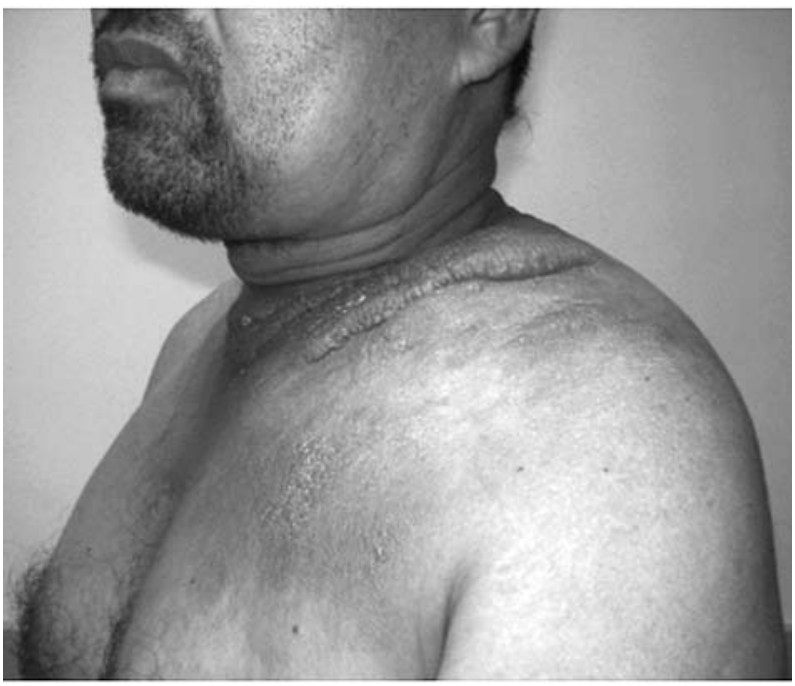

Figure 2. Photograph showing erythematous,verrucous skin over left shoulder \& clavicle 


\section{CASE REPORTS}

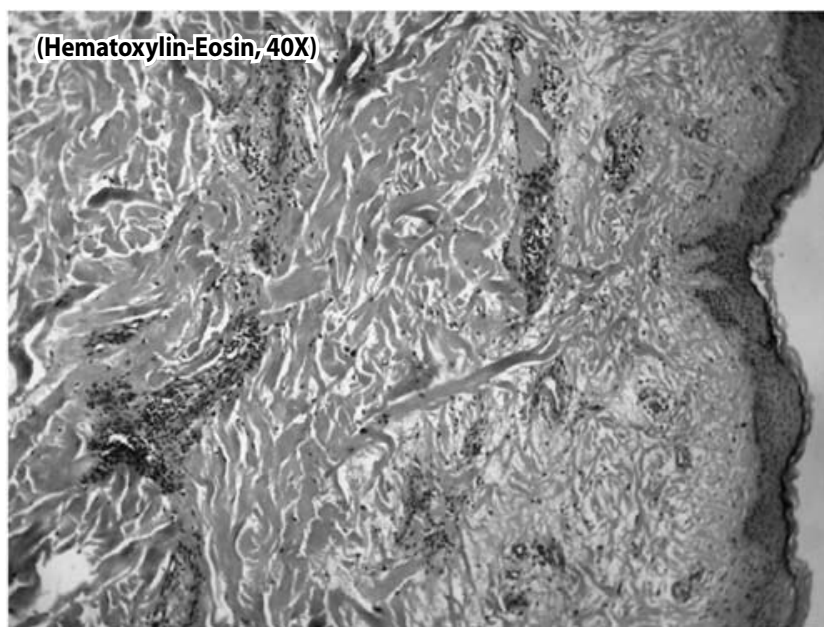

Figure 3. Histopathologic section, Hematoxylin and Eosin, low -power magnification (40x) showing a few nodular collections of cells, most hyperplastic and in mitosis, surrounded by moderately dense perivascular infiltrates of lymphocytes in the upper and deep dermis. The report stated that the epidermis was normal.

confluent nodes in levels 4 and 7 of the cervical chain and fat stranding (lymphedema) of the left supraclavicular region (Figure 4).

The patient then underwent repeat radiotherapy to the left supraclavicular, mid- upper chest and mid-upper back areas, via anteroposterior fields receiving $54 \mathrm{~Gy}$ over six weeks. Midway through the radiotherapy course, there was obvious regression of the skin lesion no longer crossing the midline. At the end of the radiotherapy course, there was even greater decrease in the skin lesion in the left supraclavicular area. However, there was edema of the left axillary area.

When he followed up after one month, there was marked improvement in the left supraclavicular area but with note of an enlarged, partially fixed left axillary lymph node. He was advised chemotherapy but opted for radiotherapy to the affected area of the axilla instead. He received 300 cGy per day for 12 days with a bioequivalent dose of $3900 \mathrm{cGy}$.

At the end of the 12 days, there was a decrease in size and improvement in mobility of the axillary node. He was referred once more for chemotherapy.

\section{DISCUSSION}

It is fairly common for a nasopharyngeal carcinoma to metastasize to the cervical lymph nodes. Often, it is the cervical lymphadenopathy that will cause patients to seek medical management. Likewise, it is common for a nasopharyngeal carcinoma to metastasize to bone. However, it is not common for nasopharyngeal cancers to metastasize to the skin.

Dermal metastasis is so termed because of the presence of metastatic tumor cells in the dermis. The epidermis is usually spared. This is most often seen in cases of breast carcinoma and is also known

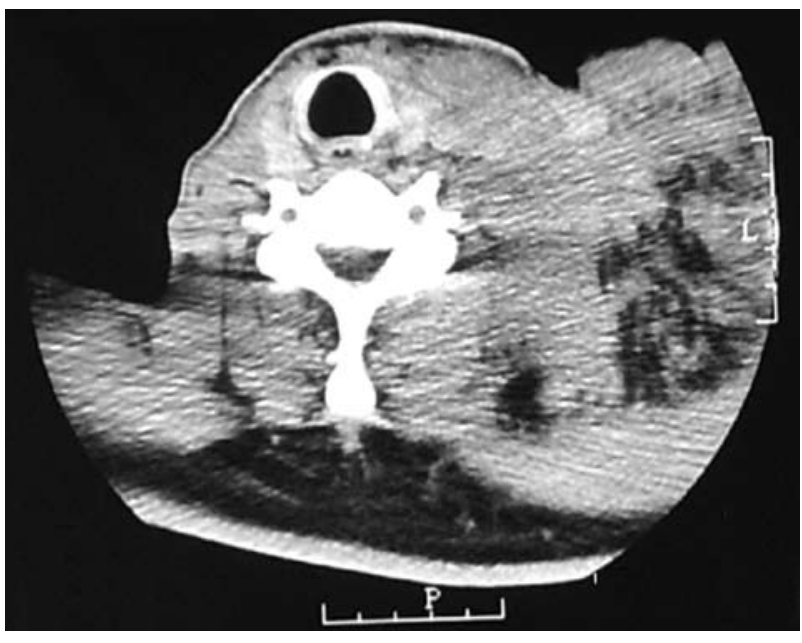

Figure 4. CT Scan showing cervical lymphadenopathy and lymphedema in November, 2005

as inflammatory carcinoma. The most frequent internal primaries that metastasize to the skin are breast, lung and gastrointestinal tract malignancies. ${ }^{4}$

A PubMed MEDLINE and Google search using the terms "nasopharynx metastasis," "dermal metastasis" and "skin metastasis"revealed only five other cases of dermal metastasis from nasopharyngeal carcinoma. Most of the articles found were for other primary cancers. Two other case reports by Dasmajundar ${ }^{5}$ and Walvekar ${ }^{6}$ cite tonsillar primaries that presented with nodular skin metastasis while Osborne reported a case of dermal metastasis from tongue carcinoma. ${ }^{7}$

Leong $^{8}$ cited three cases of recurrent nasopharyngeal carcinoma with dermal metastasis, however, all presented with facial swelling and a nodular skin lesion. Guberman ${ }^{9}$ reported a case that was similar in presentation to Leong's. Caloglu'likewise presented a case of nasopharyngeal primary with extensive nodular metastasis.

None presented like our patient-with a creeping, spreading form of dermal metastasis. Our patient presented much like a breast cancer patient would have-with skin thickening and erythema. These occurred in an area that was previously irradiated. In both Guberman's ${ }^{9}$ case and this one, there was a quandary about whether the patient had radiodermatitis or skin metastasis. In such situations, a biopsy is unavoidable and indeed, mandatory.

Our patient's dermal metastasis was treated with radiotherapy and it resolved, albeit partially. He was still referred for chemotherapy to better control his metastasis. At the end of treatment, it was fully expected that the skin metastasis would progress because of the behavior that it had 
CASE REPORTS

exhibited previously. Similar to all forms of metastasis, the prognosis is most likely poor. As of three months after the radiotherapy, the patient had not presented himself for chemotherapy.

Standard texts do not discuss dermal metastasis and thus no standard treatment options are available. To the best of our knowledge, this is to date the only reported case of dermal metastasis that was treated with radiotherapy as a single modality. As such, optimal radiotherapy technique and dosing are unknown.

Although exceedingly rare, dermal metastasis from nasopharyngeal carcinoma does happen. It does not present with pathognomonic symptomatology and therefore may be confused for a benign side effect (dermatitis), not the malignant manifestation that forebodes a bad prognosis. Patients with dermal metastasis should receive treatment, of which radiotherapy may play a significant part. Chemotherapy, though not administered in this patient, may play a role in the management of this kind of metastasis. 